\title{
A novel Streptomyces spp. integration vector derived from the S. venezuelae phage, SV1
}

\author{
Bahgat Fayed ${ }^{1}$, Ellen Younger ${ }^{2}$, Gabrielle Taylor ${ }^{1}$ and Margaret C M Smith ${ }^{1 *}$
}

\begin{abstract}
Background: Integrating vectors based on the int/attP loci of temperate phages are convenient and used widely, particularly for cloning genes in Streptomyces spp.

Results: We have constructed and tested a novel integrating vector based on g27, encoding integrase, and attP site from the phage, SV1. This plasmid, pBF3 integrates efficiently in S. coelicolor and S. lividans but surprisingly fails to generate stable integrants in S. venezuelae, the natural host for phage SV1.
\end{abstract}

Conclusion: $\mathrm{PBF} 3$ promises to be a useful addition to the range of integrating vectors currently available for Streptomyces molecular genetics.

Keywords: Streptomyces, Cloning, Integration vector, Serine integrase, Bacteriophage, SV1

\section{Background}

Bacteria in the genus Streptomyces are a prolific source of natural products, many of which are used in the clinic as antibiotic, anticancer, immune-modulatory or other therapeutic agents. Furthermore these soil bacteria have an unusual life style; vegetative growth is mycelial and when nutrients become scarce a sporulation cycle initiates [1]. The phages that infect these bacteria have been exploited in the development of vectors for genetic engineering of Streptomyces and closely related genera, in particular in the study of natural product pathways [2].

The development of integrating vectors that integrate via site-specific recombination between a site on the plasmid vector, the attP site and a site in the bacterial chromosome, the attB site have been widely adopted by researchers wishing to genetically manipulate Streptomyces genes [3]. The int/attP site from the integrating plasmid, pSAM2, was first exploited in a novel vector that could integrate into the endogenous attB site in the Streptomyces genome [4]. The advantage of the integration vectors over freely replicating plasmid vectors are the very low copy number (usually single or two copies integrated in tandem), the ease of construction of plasmids, which can be done in E. coli, and the simple method of plasmid transfer into Streptomyces via conjugation [5].

\footnotetext{
* Correspondence: maggie.smith@york.ac.uk

${ }^{1}$ Department of Biology, University of York, York YO10 5DD, UK

Full list of author information is available at the end of the article
}

The idea of using phage integrases by the research group at Eli Lilley, led to the development of integrating vectors encoding the int/attP locus from the Streptomyces phage $\phi C 31$ [6-8]. The recombination event that leads to phage integration is a conservative reciprocal DNA cleavage and rejoining mechanism occurring at the centre of the attP and $a t t B$ sites producing the integrated plasmid flanked by hybrid $a t t P / B$ sites called attL and attR [7]. Phage integrases are known to be highly directional, with tight control over integration versus excision. Integration (or $a t t B x a t t P$ ) is the default reaction for phage integrases whilst excision (attL $x$ attR) requires activation by a recombination directionality factor (RDF) or Xis $[9,10]$. Consequently the integration vectors based on the $\phi C 31$ int/attP system and lacking any other phage genes are 100\% stable in most Streptomyces species. The $\phi C 31$ integrating vectors integrated with higher efficiency and were more stable than the pSAM2-derived integration vectors and are now widely adopted by researchers in Streptomyces genetics. The use of the $\phi C 31$ integration system is also being widely adopted for genome engineering in eukaryotes, in particular in tissue culture and model organisms such as the mouse and Drosophila [11].

In 2003 the int/attP locus from the phage $\phi B T 1$ was used to generate an alternative suite of phage-derived integration vectors for Streptomyces [12]. These vectors were demonstrated to be completely orthogonal to the $\phi C 31$ derived plasmids and plasmids derived from the 
two phage int/attP loci could be used in combination without loss of integrating efficiency. The $\phi B T 1$ int/attP integration vectors are also widely used in the Streptomyces community. Recently vectors based on phage TG1 have been developed for use in Streptomyces avermitilis [13].

Here we present a new integrating vector derived from $S$. venezuelae phage SV1 $[14,15]$. We demonstrate the efficient integration of the plasmid into several Streptomyces spp. although, surprisingly, we failed to obtain stable integrants in $S$. venezuelae.

\section{Results and discussion}

The genome of phage SV1 was sequenced previously and, consistent with the temperate nature of the phage, g27 encodes an integrase [15]. Gp27 is a serine integrase, whose closest homologue in the database is from Streptomyces prunicolor (WP_019054986.1; 54\% identity). SV1 is only distantly related (between 11 and 13\% identical) to $\phi C 31, T G 1$ and $\phi B T 1$ integrases so the SV1 integration system encoded by SV1 g27/attP is therefore very likely to be another orthologous system to $\phi \mathrm{C} 31$ and $\phi \mathrm{BT} 1$ integration systems.

The DNA upstream and downstream of SV1 g27 was studied for a likely attP site. Precedent dictates that attP is normally upstream or downstream of the integrase gene but in some mobile genetic elements, such as SCCmec, can be located quite distal from their cognate integrase genes $[11,16]$. In SV1 the attP site is unlikely to be upstream of $g 27$ as the upstream gene, $g 28$, overlaps with $g 27$ by the sequence 5'ATGA, which couples the start codon (ATG) of $g 27$ with the stop codon of $g 28$ (TGA). Downstream of $g 27$ is a non-coding region of 342 bp before the start of the downstream gene, $g 26$. The attP sites for the serine integrases commonly comprise a perfect inverted repeat flanking a spacer of at least $20 \mathrm{bp}$ [11]. Within the g26-g27 intergenic region in SV1 there are two perfect inverted repeats (IRs); the IR distal to $g 27$ has a spacer of $5 \mathrm{bp}$ and the IR proximal to $g 27$ has a 22 bp spacer. Moreover the IR proximal to $g 27$ has a $10 \mathrm{bp}$ perfect inverted repeat so, together with the spacer DNA, the length of this DNA element is $42 \mathrm{bp}$ which is the same length as the $\phi C 31$ attP site. The length attP sites used by other serine integrases is between 42 and $69 \mathrm{bp}[11,17]$. The attP site in SV1 is therefore likely to be located between nucleotides 20504 and 20545 and is downstream of the integrase gene, $g 27$.

As the SV1 g27 is likely to be expressed as part of an operon, we decided to test its integrating properties by swapping the $\phi C 31$ int ORF expressed from the tcp830 promoter in pEY25 for the SV1 g27/attP locus (Figure 1). Primers were designed to clone the SV1 g27/attP locus 20487 to 22295 comprising the putative attP site and the $g 27$ ORF in both orientations downstream of the tcp830 promoter in pEY25, to generate pMS98 and
pBF1 (Figure 1). Both plasmids were introduced into E. coli ET12567 (pUZ8002) and used in conjugation reactions with S. coelicolor J1929. Surprisingly the numbers of exconjugants were not significantly different for pBF1 (in which the SV1 $g 27$ is co-directional with the tcp830 promoter) and pMS98 (in which the SV1 g27 is oriented towards tcp830) and this occurred with or without addition of anhydrotetracycline (Table 1). In fact exconjugants containing pBF1 tended to overproduce the two pigments actinorhodin and undecylprodigiosin indicating a possible stress response, perhaps due to overexpression of SV1 integrase. We assume that there are fortuitous sequences upstream of the $g 27$ gene in pMS98 that resemble both promoter and ribosome binding sites for integrase expression after conjugation to Streptomyces. The low frequency of exconjugants from $E$. coli containing pEY25 in this experiment is likely to be due to the absence of the $\phi C 31$ attP site (Figure 1).

To validate the integration of the plasmids via SV1 g27/attP site into the $S$. coelicolor genome, we have identified the integration site of SV1 phage using an inverse PCR technique. Genomic DNA from an exconjugant of S. coelicolor J1929 containing the integrated pMS98 was cut with a restriction enzyme for which there is no recognition site within pMS98 and self-ligated. Primers reading outwards from the SV1 plasmid into the S. coelicolor $J 1929$ genome were then used to generate a PCR product (Figure 2). The plasmid pMS98 was found to have integrated into SCO4383 encoding a putative 4-CoumarateCoA Ligase, a key enzyme in the phenylpropanoid pathway that, at least in plants, is important in secondary metabolism pathways for flavonoids and monolignols [18]. Based on the DNA sequence of this PCR product, we constructed two further primers against the integration region (SC04383) to amplify attB, attR, and attL (using S. coelicolor DNA, and S. coelicolor J1929:pMS98 genomic DNA as templates) (Figure 3). The resulting DNA sequences confirmed that the SV1 attB site is within SCO4383 and the attP site is located, as predicted, downstream of the SV1 g27 gene (Figure 4). The attP and the $a t t B$ have similar features to those observed in the other serine integrase attachment sites; they both contain inverted repeats but these are different in the $a t t B$ and attP sequences, and there is an identical sequence of $4 \mathrm{bp}$ in the centre of both substrates where the recombination occurs (Figure 4) [11,12].

Plasmid pMS98 was modified to remove unnecessary DNA, the attR site from $\phi C 31$, and the tcp830 promoter to generate $\mathrm{pBF} 3$ (Figure 1). This novel integration vector has unique XhoI, XbaI, KpnI and PvuII sites for cloning. To test whether pBF3 could integrate into a range of Streptomyces genomes it was introduced by conjugation into S. coelicolor, S. lividans, S. venezuelae, $S$. avermitilis and $S$. albus. Conjugation frequencies of 


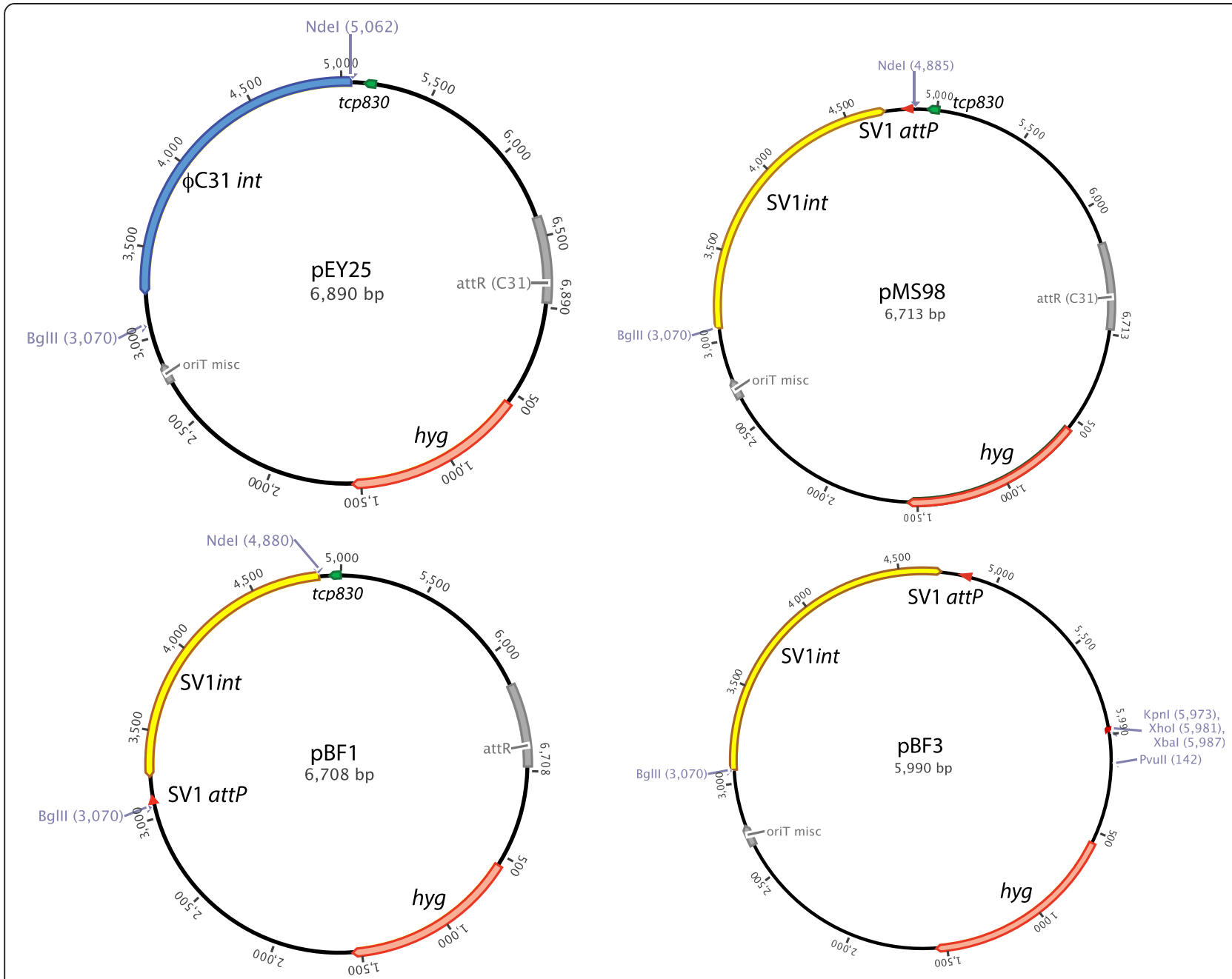

Figure 1 Plasmids constructed during the course of this work. pMS98 and pBF1 are derivatives of the plasmid pEY25, in which the $\phi C 31$ int gene (blue arrow) is replaced with the SV1 g27 (encoding SV1 integrase; yellow arrow) and attP (red arrow) in orientations in which the SV1 g27 genes is being expressed from the tcp830 promoter (pBF1) or is orientated opposite to the tcp830 promoter (pMS98). pBF3 is a derivative of PMS98 in which the tcp830 promoter and the $\$ C 31$ attR sites have been removed. The hygromycin resistance gene is represented by the pale red arrow and the tcp 830 promoter by the green arrow. oriT is in grey.

pBF3 into S. coelicolor and S. lividans were reminiscent of those obtained with $\phi \mathrm{BT} 1$ and $\phi C 31$ integration plasmids; the numbers of hygromycin resistant exconjugants for S. coelicolor J1929, S. coelicolor M512, and S. lividans TK24 were greater than $1 \times 10^{5}$ (Table 2), and the integrations were stable after two rounds of sporulation without selection (see below). When S. venezuelae was used as the recipient, the hygromycin resistant exconjugants were, although numerous, very small and the integrations were not stable as after two rounds of sporulation, hygromycin resistance was lost. A BLAST search using the SV1 attB site from S. coelicolor revealed that highly similar sequences were indeed present in $S$. lividans and $S$. avermilitis but no homologous sequences were found in $S$. albus or S. venezuelae. Despite the presence of a putative attB site for SV1 in S. avermitilis the frequency of conjugation and integration of pBF3 was very low (Table 2).

The sequences of the SV1 attB and attP sites are distinct from the recombination sites for the other known phage integrases. We showed previously that integrating vectors derived using integrases from $\phi \mathrm{C} 31$ and $\phi \mathrm{BT} 1$ do not interfere with each other with respect to the frequency of integration or their stability [12]. We therefore tested whether the integration frequencies of $\phi C 31$ or $\phi B T 1$ derived integrating vectors were affected if the recipient already contained pBF3 integrated at the SV1 attB site. Conjugations were performed using E. coli donors containing either pSET152 (encoding $\phi C 31 \mathrm{int} / \mathrm{attP}$ ) or pRT801 (encoding $\phi B T 1$ int/attP), both plasmids conferring apramycin resistance, and S. coelicolor M512 containing pBF3 as recipient. Selection was for both hygromycin 
Table 1 Conjugation frequnecies of various integrating plasmids into S. coelicolor J1929

\begin{tabular}{lcc}
\hline $\begin{array}{l}\text { E. coli ET12567 } \\
\text { (pUZ8002) donor } \\
\text { containing: }\end{array}$ & $\begin{array}{c}\text { Origin of integrase } \\
\text { and attP }\end{array}$ & $\begin{array}{c}\text { Hygromycin resistant } \\
\text { exconjugants/10 } \\
\text { spores }\end{array}$ \\
\hline pMS98 & SV1 & $2.4 \times 10^{6}$ \\
pBF1 & SV1 & $1.1 \times 10^{7}$ \\
pBF1 $^{*}$ & SV1 & $9.9 \times 10^{6}$ \\
pBF3 & SV1 & $1.4 \times 10^{7}$ \\
pEY25 & $\phi C 31$ (int only) & $9 \times 10^{3}$ \\
\hline
\end{tabular}

*The SV1 integrase was induced with $1 \mu \mathrm{g} / \mathrm{ml}$ anhydrotetracycline.

and apramycin. There was no great reduction in the conjugation frequency compared with the use of plasmid-free S. coelicolor M512 as a recipient (Table 2). SV1 vectors can therefore be used in combination without interference with $\phi C 31$ and $\phi$ BT1 derived vectors.

Hygromycin resistant colonies obtained after conjugation of E. coli containing pBF3 with S. coelicolor J1929, S. coelicolor M512, S. lividans TK24 and S. venezuelae were allowed to sporulate and were subcultured twice on medium without selection. Genomic DNA was isolated and analysed by Southern blotting (Figure 5). The expected $4.3 \mathrm{kbp}$ band, indicative of integrated pBF3, was observed in the DNA from S. coelicolor and S. lividans exconjugants. The absence of the $4.3 \mathrm{kbp}$ band from the $S$. venezuelae genomic DNA indicated that pBF3 did not persist in this strain and was lost. Stable hygromycin resistant $S$. venezuelae exconjugants were obtained with the $\phi B T 1$ and $\phi C 31$ derived vectors (pMS82 and $\phi C 31$, respectively; Table 2). Paradoxically a lysogen of SV1 in $S$. venezuelae is perfectly stable and grows like the nonlysogen. We deduce that the interruption in SCO4383 caused by the integrating plasmid is toxic, but the toxicity is ameliorated by a prophage-encoded gene or by an unknown cis effect within the integrated prophage. Notably SV1 does not encode a homologue to SCO4383, or fragments of SCO4383 that could compensate for its truncation by integration of SV1 derived integrating vectors.

\section{Conclusions}

The activity of a novel phage integration system from bacteriophage SV1 has been demonstrated in S. coelicolor and $S$. lividans and the $a t t P$ and $a t t B$ sites identified. We believe that the new integrating vector pBF3 will be of use in the genetic manipulation of these and other Streptomyces strains. More generally the characterization of a new integrase and its substrates will provide biologists with new tools for DNA assembly in the genomes of a wide range of microorganisms and other model organisms.

\section{Methods}

\section{Bacterial strains and culture}

E. coli strain DH5 $\alpha$ was used for plasmid construction. E. coli strain ET12567 (pUZ8002) is a methylationdefective strain (dam-13: Tn9 dcm-6 hsdM) and was used as the conjugation donor in plasmid conjugations from E. coli to Streptomyces [20].

Six Streptomyces strains were used as recipients for intergeneric conjugation: Streptomyces coelicolor J1929 (contains $\Delta p g l Y$ conferring sensitivity to $\phi C 31$ and $\phi B T 1$;

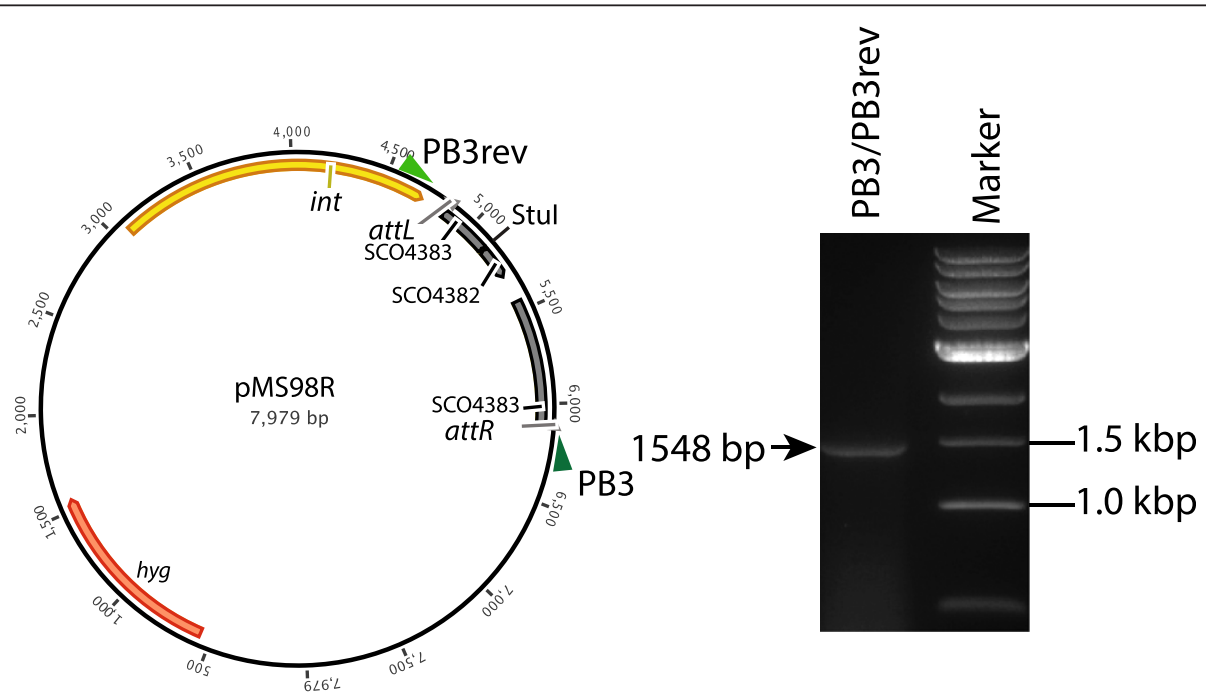

Figure 2 Rescue of the integrated plasmid and determination of the sequence of attB. The structure of the rescued plasmid, pMS98R, by digestion of genomic DNA from an S. coelicolor 1929 pMS98 exconjugant with Stul and self-ligation. The two primers PB3 and PB3rev were used to amplify the DNA reading out from the attL and attR sites produced on integration of pMS98 into the attB site. The PCR amplified DNA generated was separated by electrophoresis on a $0.8 \%$ agarose gel. The size of the band obtained is in agreement with the predicted 1548 bp fragment, after performing the manipulations in silico using the published S. coelicolor genome sequence [19]. 


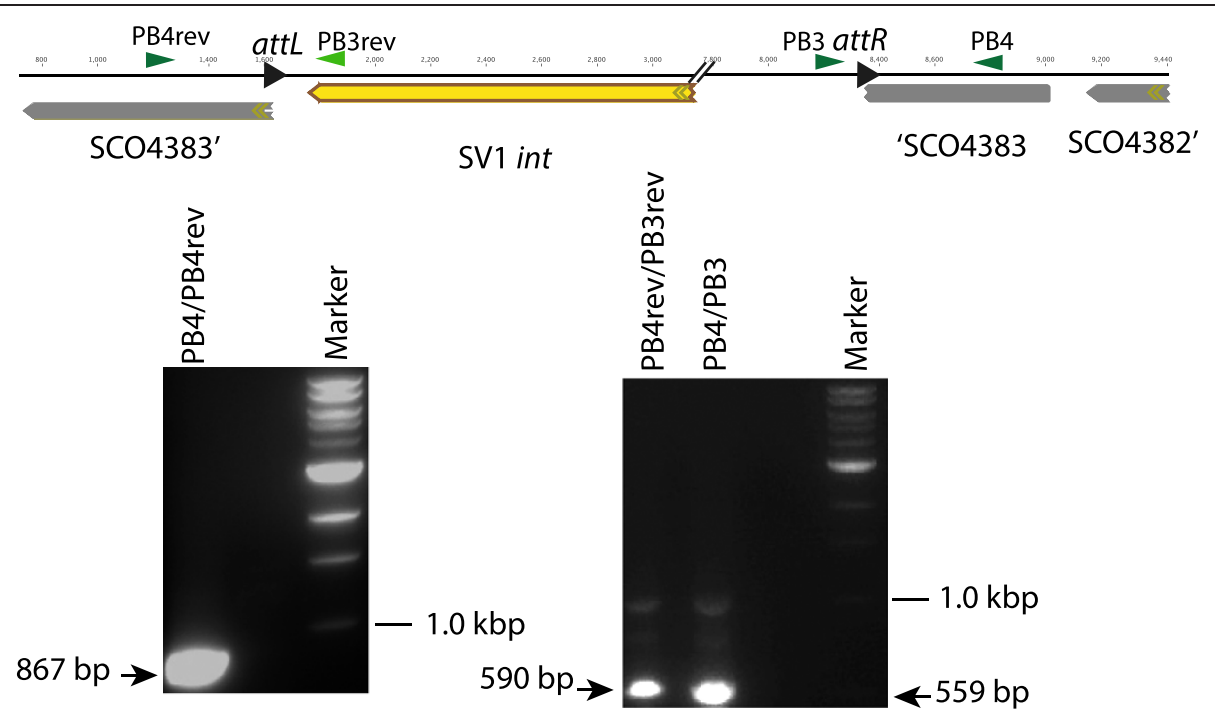

Figure 3 Validation of the position of the integrated plasmid, pMS98, in the S. coelicolor genome. Primers PB4 and PB4rev were designed to flank the SV1 attB site and were predicted to amplify a 867 bp fragment as shown in the agarose gel depicted on the left. Using pairs of primers PB4rev with PB3rev and PB3 with PB4, the attL and attR sites were amplified from S. coelicolor J1929:pMS98 genomic DNA. Fragments of the predicted sizes were obtained as depicted in the agarose gel (right).

[21]) Streptomyces coelicolor M512 (AredD $\triangle$ actII-ORF4 $\mathrm{SCP}^{-} \mathrm{SCP}^{-} \mathrm{Pgl}^{+}$) [22], Streptomyces avermitilis MA4680 [23], Streptomyces venezuelae 10712 [24], Streptomyces albus J1074 [25], Streptomyces lividans TK24 (str-6 SLP2-, SLP3 ${ }^{-}$)[26].

The E. coli strains DH5 [27] and ET12567(pUZ8002) $[20,26]$ were grown in Luria-Bertani broth (LB) or on LB agar at $37^{\circ} \mathrm{C}$. Streptomyces strains were grown in Soya Mannitol (SM) agar at $30^{\circ} \mathrm{C}$ for routine maintenance [26]. Conjugations were performed on SM containing $10 \mathrm{mM}$ $\mathrm{MgCl}_{2}$ and Yeast extract malt extract medium was used for the preparation of genomic DNA [26]. Antibiotic concentrations for $E$. coli were $150 \mu \mathrm{g} / \mathrm{ml}$ hygromycin, $50 \mu \mathrm{g} / \mathrm{ml}$ apramycin, $50 \mu \mathrm{g} / \mathrm{ml}$ kanamycin, $25 \mu \mathrm{g} / \mathrm{ml}$ chloramphenicol and $100 \mu \mathrm{g} / \mathrm{ml}$ hygromycin, $50 \mu \mathrm{g} / \mathrm{ml}$ apramycin and $25 \mu \mathrm{g} / \mathrm{ml}$ nalidixic acid for selection with Streptomyces.

\section{DNA manipulation}

Plasmids preparations, E. coli transformations, DNA digestion by restriction enzymes, DNA fragment isolation and purification, and gel electrophoresis were carried

A

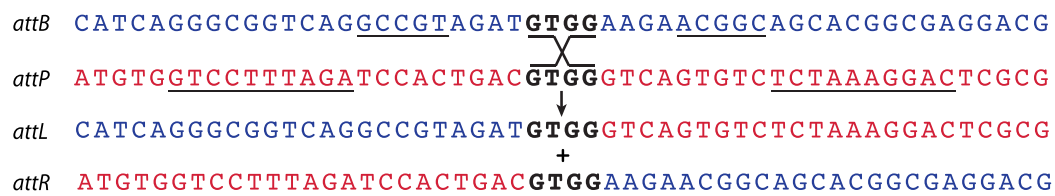

B

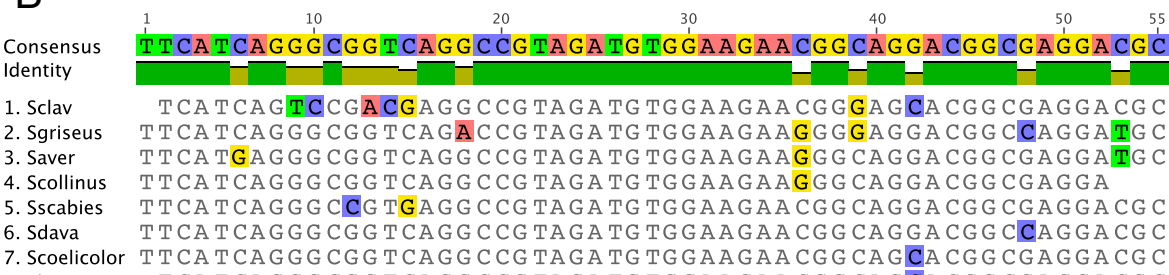

7. Scoelicolor T TCA TCA G G CGG TCA G C CG TAGA TG TGGAAGAA CG GA GCA CGGCGAGGACG C

8. Sliv TCA TCAGGGCGG TCAGGCCGTAGATG TGGAAGAA CG CA CA CG CGAGGACGC

Figure 4 Sequences of the $a t t B$, attP, attL and attR sites used by SV1 integrase. Panel $\mathbf{A}$. The crossover site occurs within the 4 bp sequence in black and the positions of the inverted repeats are underlined in the attP and attB sites. Panel $\mathbf{B}$. The results of a BLAST search for the SV1 attB site in Streptomyces genomes deposited in the nucleotide sequence database. The default settings did not identify an SV1 attB site from S. venezuelae or S. albus. 
Table 2 Conjugation frequencies per $10^{8}$ spores of integrating plasmids into Streptomyces species

\begin{tabular}{|c|c|c|c|c|}
\hline \multirow[t]{2}{*}{ Streptomyces recipient: } & \multicolumn{4}{|c|}{ E. coli donor, ET12567 (pUZ8002) containing plasmids: } \\
\hline & pBF3 (SV1 g27/attP) & pMS82 ( & pSET152 ( $($ C31 int/attP) & pRT801 ( $(\mathrm{BT} 1$ int/attP) \\
\hline S. coelicolor J1929 & $2.5 \times 10^{6}$ & $3.6 \times 10^{6}$ & $6 \times 10^{5}$ & $N D^{1}$ \\
\hline S. coelicolor M512 & $1.7 \times 10^{7}$ & $6 \times 10^{7}$ & $6 \times 10^{6}$ & ND \\
\hline S. lividans & $1.4 \times 10^{5}$ & $9.5 \times 10^{5}$ & $2.3 \times 10^{5}$ & ND \\
\hline S. venezuelae 10712 & $3.3 \times 10^{4}$ & $1.7 \times 10^{7}$ & $9.8 \times 10^{4}$ & ND \\
\hline S. avermitilis & 40 & $3 \times 10^{5}$ & $1.8 \times 10^{3}$ & ND \\
\hline S. albus J1074 & $4.6 \times 10^{2}$ & $5 \times 10^{5}$ & $1 \times 10^{3}$ & ND \\
\hline S. coelicolor M512: pBF3 & - & - & $4 \times 10^{6}$ & $2.8 \times 10^{5}$ \\
\hline
\end{tabular}

${ }^{1}$ Not Done.

out according to Sambrook et al. [27]. In-Fusion ${ }^{\bullet}$ cloning $\left(\right.$ Clontech $^{\odot}$ ) was generally used for joining DNA fragments. DNA preparation from Streptomyces was performed following the Streptomyces manual [26].

Southern blotting was performed, according to the manufacturers instructions, on Hybond-N nylon membrane (Amersham) using a fragment of DNA derived from the hygromycin resistant gene as the probe. The AlkPhos Direct Labeling and Detection System with CDP-Star kit (Amersham) was used for detection. $1 \mu \mathrm{g}$ of NruI (New England Biolabs) digested genomic DNA was loaded onto a $0.8 \%$ agarose gel in TBE buffer and electrophoreses overnight prior to capillary blotting.

Polymerase Chain Reaction (PCR) was carried out using Phusion ${ }^{\bullet}$ High-Fidelity DNA Polymerase (New England Biolabs) according to the manufacturer's instructions.

\section{Plasmid constructions}

pEY25 is a derivative of pAV11, an integration vector that encodes the $\phi B T 1$ int/attP locus and the anhydrotetracycline inducible promoter, tcp830. To generate pEY25 the $\phi B T 1$ int gene was deleted and the $\phi C 31$ int gene was placed under the control of the tcp830 promoter. pMS98 was constructed by PCR amplification of SV1 g27/ attP locus using primers MS409 (5' GCTTCATATGAAA
CGAGACCTACCAAG) and MS410 (5'CGTTAGATCT TCGCGCTCCGATGTGGTC) and In-Fusion ${ }^{\oplus}$ cloning into pEY25 cut with NdeI and BglII to replace the $\phi C 31$ int gene. pBF1 was constructed in the same way but using primers PBF1for (5' AAGGAGATATACATATGAAACG AGACCTACCAAGC- 3') and PBF1rev (5' CCATGAG CCAAGATCTTCGCGCTCCGATGTGGTCC- 3'). pBF3 was constructed as follows to remove unnecessary elements of pBF1: pBF1 was first cut with AvrII, and Acc65I, and the ends filled in with DNA Polymerase I, Large (Klenow) Fragment (New England Biolabs) to generate blunt ends for ligation. This blunt ended fragment was then self-ligated using Quick ligase enzyme (New England Biolabs) to produce pBF2. To remove the tcp 830 promoter, pBF2 was digested with NdeI, and AseI and the 5985 bp fragment was self-ligated to form $\mathrm{pBF}$.

\section{Inverse PCR}

Inverse PCR was performed to identify the integration site of SV1 within Streptomyces coelicolor J1929. This procedure is designed for amplifying anonymous flanking genomic DNA regions. Genomic DNA was prepared from a strain containing the integrated plasmid, pMS98, digested with an enzyme that does not cut within the plasmid (StuI) and then ligation of DNA under dilute

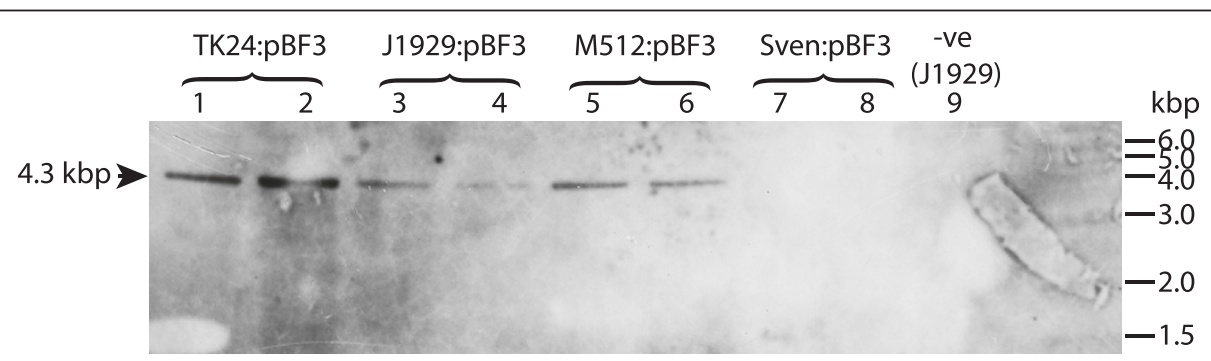

Figure 5 Southern blot to demonstrate the presence of the predicted hygromycin gene fragment in S. coelicolor and S. lividans exconjugants. Two independent exconjugants derived from an E. coli donor containing pBF3 and S. coelicolor, S. lividans and S. venezuelae as recipients were initially selected using the hygromycin resistance marker on pBF3 but then subsequently maintained without selection. Genomic DNA was then prepared from each line. The hyg gene was detected in S. coelicolor:pBF3 and S. lividans:pBF3 lines but not in the S. venezuelae lines indicating that pBF3 is not stably maintined in S. venezuelae. 
DNA conditions to favour circularization. Finally, PCR amplification was performed using oligonucleotides PB3 for (5' GTACGTCGGAGGTCTAGAGA) and PB3rev (5' GCAGCTTCGAGTTTCATCCCG) that prime DNA synthesis from the known sequence within pMS98. To confirm the SV1 integration site, primers $\mathrm{PB} 4$ for $\left(5^{\prime} \mathrm{CA}\right.$ CAGCCCCAACACCGTC) and PB4 rev (5' -GTCGG TGAGGGAGACGATG) were designed to amplify the potential SV1 attB from the S. coelicolor J1929 DNA. These primers were also used with PB3 and PB3rev to amplify the potential attR, attL from the exconjugants $S$. coelicolor J1929 pMS98 DNA.

\section{Competing interests}

The authors declare that they have no competing interests.

\section{Authors' contributions}

$\mathrm{BF}$ designed and performed the experiments and helped to write the paper. EY and GT designed and performed experiments. MCMS designed experiments, raised funding and helped to write the paper. All authors read and approved the final manuscript.

\section{Acknowledgements}

We are grateful to Dr Maureen Bibb for providing SV1, S. venezuelae, the S. venezuelae SV1 lysogen, to Professor Mark Buttner for S. coelicolor strain J1929, to Professor Mervyn Bibb for S. coelicolor M512 and E. coli strain ET12567(pUZ8002), to Professor Keith Chater for S. albus J1074 and to the late Professor Simon Baumberg for S. avermitilis. We are also grateful to Dr Maureen Bibb for discussions on the manuscript. BF acknowledges receipt of a PhD studentship from the Egyptian Ministry of Higher Education and additional funding for his internship at the University of York. This work was funded by the Biotechnology and Biological Science Research Council, UK grant references BB/K003356 and BB/H001212.

\section{Author details}

${ }^{1}$ Department of Biology, University of York, York YO10 5DD, UK. ${ }^{2}$ School of Medical Sciences, Institute of Medical Sciences, University of Aberdeen, Aberdeen AB25 2ZD, UK

\section{Received: 6 January 2014 Accepted: 12 May 2014}

Published: 30 May 2014

\section{References}

1. Chater KF: Streptomyces inside-out: a new perspective on the bacteria that provide us with antibiotics. Philos Trans R Soc Lond B Biol Sci 2006, 361(1469):761-768.

2. Baltz RH: Strain improvement in actinomycetes in the postgenomic era. $J$ Ind Microbiol Biotech 2011, 38(6):657-666.

3. Baltz RH: Streptomyces temperate bacteriophage integration systems for stable genetic engineering of actinomycetes (and other organisms). J Ind Microbiol Biotech 2012, 39(5):661-672.

4. Boccard F, Smokvina T, Pernodet JL, Friedmann A, Guerineau M: Structural analysis of loci involved in PSAM2 site-specific integration in Streptomyces. Plasmid 1989, 21(1):59-70.

5. Smokvina T, Mazodier P, Boccard F, Thompson CJ, Guerineau M: Construction of a series of pSAM2-based integrative vectors for use in actinomycetes. Gene 1990, 94(1):53-59.

6. Bierman M, Logan R, O'Brien K, Seno ET, Rao RN, Schoner BE: Plasmid cloning vectors for the conjugal transfer of DNA from Escherichia coli to Streptomyces spp. Gene 1992, 116(1):43-49.

7. Kuhstoss S, Rao RN: Analysis of the integration function of the streptomycete bacteriophage $\phi$ C31. J Mol Biol 1991, 222(4):897-908.

8. Kuhstoss S, Richardson MA, Rao RN: Plasmid cloning vectors that integrate site-specifically in Streptomyces spp. Gene 1991, 97(1):143-146.

9. Khaleel T, Younger E, McEwan AR, Varghese AS, Smith MCM: A phage protein that binds $\phi C 31$ integrase to switch its directionality. Mol Microbiol 2011, 80(6):1450-1463.
10. Lewis JA, Hatfull GF: Control of directionality in integrase-mediated recombination: examination of recombination directionality factors (RDFs) including Xis and Cox proteins. Nucl Acid Res 2001, 29(11):2205-2216.

11. Brown WR, Lee NC, Xu Z, Smith MCM: Serine recombinases as tools for genome engineering. Methods 2011, 53(4):372-379.

12. Gregory MA, Till R, Smith MCM: Integration site for Streptomyces phage $\phi \mathrm{BT} 1$ and the development of novel site-specific integrating vectors. $J$ Bacteriol 2003, 185(17):5320-5323.

13. Morita K, Yamamoto T, Fusada N, Komatsu M, Ikeda H, Hirano N, Takahashi H: The site-specific recombination system of actinophage TG1. FEMS Microbiol Lett 2009, 297(2):234-240.

14. Stuttard C: Generalized transduction in Streptomyces species. In Genetics and Molecular Biology of Industrial Microorganisms. Edited by Hershberger CL, Queener SW, Hegeman G. Washington DC: Amercian Society of Microbiology; 1989:157-162.

15. Smith MCM, Hendrix RW, Dedrick R, Mitchell K, Ko CC, Russell D, Bell E, Gregory M, Bibb MJ, Pethick F, Jacobs-Sera D, Herron P, Buttner MJ, Hatfull G: Evolutionary relationships among actinophages and a putative adaptation for growth in Streptomyces spp. J Bacteriol 2013, 195(21):4924-4935.

16. Hanssen A-M, Sollid JUE: SCCmec in staphylococci: genes on the move. FEMS Immunol Med Microbiol 2005, 46:8-20.

17. Xu Z, Thomas L, Davies B, Chalmers R, Smith MCM, Brown WRA: Accuracy and efficiency define $B \times b 1$ integrase as the best of fifteen candidate serine recombinases for the integration of DNA into the human genome. BMC Biotech 2013, 13:87.

18. Santos CN, Koffas M, Stephanopoulos G: Optimization of a heterologous pathway for the production of flavonoids from glucose. Metab Eng 2011, 13(4):392-400.

19. Bentley SD, Chater KF, Cerdeno-Tarraga AM, Challis GL, Thomson NR, James KD, Harris DE, Quail MA, Kieser H, Harper D, Bateman A, Brown S, Chandra G, Chen CW, Collins M, Cronin A, Fraser A, Goble A, Hidalgo J, Hornsby T, Howarth S, Huang CH, Kieser T, Larke L, Murphy L, Oliver K, O'Neil S, Rabbinowitsch E, Rajandream MA, Rutherford K: Complete genome sequence of the model actinomycete Streptomyces coelicolor A3(2). Nature 2002, 417(6885):141-147.

20. MacNeil DJ: Characterization of a unique methyl-specific restriction system in Streptomyces avermitilis. J Bacteriol 1988, 170(12):5607-5612.

21. Bedford DJ, Laity C, Buttner MJ: Two genes involved in the phase-variable $\phi C 31$ resistance mechanism of Streptomyces coelicolor A3(2). J Bacteriol 1995, 177(16):4681-4689.

22. Floriano B, Bibb M: afsR is a pleiotropic but conditionally required regulatory gene for antibiotic production in Streptomyces coelicolor A3 (2). Mol Microbiol 1996, 21(2):385-396.

23. Ikeda H, Ishikawa J, Hanamoto A, Shinose M, Kikuchi H, Shiba T, Sakaki Y, Hattori M, Omura S: Complete genome sequence and comparative analysis of the industrial microorganism Streptomyces avermitilis. Nat Biotechnol 2003, 21(5):526-531.

24. Pullan ST, Chandra G, Bibb MJ, Merrick M: Genome-wide analysis of the role of $\mathrm{G} \ln R$ in Streptomyces venezuelae provides new insights into global nitrogen regulation in actinomycetes. BMC Genomics 2011, 12:175

25. Chater KF, Wilde LC: Streptomyces albus G mutants defective in the SalGI restriction-modification system. J Gen Microbiol 1980, 116(2):323-334.

26. Kieser T, Bibb MJ, Buttner MJ, Chater KF, Hopwood DA: Practical Streptomyces Genetics. Norwich: The John Innes Foundation; 2000.

27. Sambrook J, Russell DW: Molecular Cloning: A Laboratory manual. New York: Cold Spring Harbor Laboratory Press; 2001.

doi:10.1186/1472-6750-14-51

Cite this article as: Fayed et al:: A novel Streptomyces spp. integration vector derived from the S. venezuelae phage, SV1. BMC Biotechnology 2014 14:51. 